\title{
Feeding Strategies for Maximising Gross Margin in Pig Production
}

\author{
David L.J. Alexander ${ }^{12}$ \\ Institute of Information Sciences and Technology \\ Massey University \\ New Zealand \\ David.Alexander.3@uni.massey.ac.nz \\ Patrick C.H. Morel \\ Institute of Food, Nutrition and Human Health \\ Massey University \\ New Zealand \\ P.C.Morel@massey.ac.nz \\ Graham R. Wood ${ }^{1}$ \\ Institute of Information Sciences and Technology \\ Massey University \\ New Zealand \\ G.R.Wood@massey.ac.nz
}

\begin{abstract}
Pig farming is, by weight, the largest meat industry in the world. This paper reports on the successful application of stochastic optimisation algorithms to the problem of choosing feeding strategies to maximise gross margin. A pig growth model and a combination of optimisation techniques allow the composition and quantity of feed to be optimised so as to maximise the return to the farmer. The nature of the objective function is examined, providing a theoretical explanation of the observed level of superiority of optimisation algorithms over pure random search.
\end{abstract}

\section{Introduction}

Production of pig meat worldwide exceeds that of any other meat. Pigs are generally fed in a controlled environment, with least cost diets determined using linear programming. More important to the pig producer than using least cost diets, however, is maximising total gross margin (per pig place or per pig place per year). Optimisation of pig feeding strategies for maximum gross margin is now possible, using two relatively recent developments: computerised pig growth models, and efficient methods for optimisation of high dimensional non-linear functions. The method of solution for this problem

\footnotetext{
${ }^{1}$ Supported by the Marsden Fund administered by the Royal Society of New Zealand

${ }^{2}$ Supported by a Bright Future Scholarship administered by the Foundation for Research, Science and Technology
} 
involves a synthesis of optimisation techniques, traditional linear programming being employed as a sub-routine within the framework of a wider search algorithm. The purpose of this paper is to describe this problem, together with the solution methodology and some associated practical outcomes.

The format of the paper is as follows. In the next section the optimisation problem is formulated, the domain described and the objective function stated. Successful solution approaches are described in Section 3. The nature of the objective function is discussed in Section 4. A summary then concludes the paper.

\section{Problem Formulation}

This section defines several variables associated with pig production, and introduces the pig growth model used to simulate growth of a pig by computer. This growth model, with the use of linear programming, is the mechanism by which values of input parameters are combined to provide a measure of the overall quality of the feeding strategy they define. This measure of quality is the objective function for optimisation, defined below. The complete formulation of the problem is then ready for input into a non-linear optimisation algorithm.

\subsection{Parameters}

Parameters affecting the farming of pigs from weaning until slaughter include some external factors; the summary characteristics of the pig; and the feeding strategy that is to be administered.

External factors are the cost of purchasing the weaner, the price schedule for pigs at slaughter, and the number of days required for farm maintenance after slaughter of one pig and before the purchase of another. These parameters are needed to evaluate the objective function. The first two are outside the farmer's control; the third is observed on farm.

The characteristics of different pig genotypes are summarised, as in DeLange [1], by three variables:

$$
\begin{aligned}
& P_{0}=\text { initial mass of protein in the weaner pig } \\
& P d_{\max }=\text { maximum daily protein deposition } \\
& \text { min } L P=\text { minimum allowable lipid to protein ratio }
\end{aligned}
$$

The main area over which the pig producer has control is the feeding of the pig. A single feeding regimen can be summarised, as described in DeLange [1], by three parameters: $d, r$ and $p$. A feeding strategy $F$ is then a finite sequence of $(d, r, p)$ triples, with each triple describing the diet for a fixed period, say one week. Here

$$
\begin{aligned}
& d=\text { digestible energy density, in MegaJoules per kilogram } \\
& r=\text { minimum lysine to digestible energy ratio, in grams per MegaJoule } \\
& p=\text { proportion of the ad libitum daily digestible energy intake }
\end{aligned}
$$

The ad libitum digestible energy intake for the pig at any given liveweight is determined on farm using monitor pens of pigs. Feed supplied, feed wasted and pig liveweight are recorded in order to determine the voluntary daily feed intake of the pig. 
For the purposes of this exposition a feeding strategy of ten $(d, r, p)$ triples is used, each fed for one week, so the general point in the domain has the form

$$
F=\left(d_{1}, r_{1}, p_{1} ; d_{2}, r_{2}, p_{2} ; \ldots ; d_{10}, r_{10}, p_{10}\right)
$$

For the particular problem examined here, relevant to the New Zealand situation, a growth period of ten weeks is sufficient to include the most profitable solutions.

The energy content of the ingredients in the diet requires that $d$ be at least $12 \mathrm{MJ} / \mathrm{kg}$ and no more than $16 \mathrm{MJ} / \mathrm{kg}$. A sensible range for $r$ is from 0.4 to 1.0 grams of lysine per MegaJoule; values outside this range are feasible, but this range has been found wide enough to encompass the most profitable diets and is in accordance with the opinion of pig nutritionists. Finally, $p$ is allowed to vary between 0.5 and 1.0; nutritionists agree that pigs require at least $50 \%$ of their maximum voluntary digestible energy intake. In summary, the general point in the domain lies within $([12,16] \times[0.4,1] \times[0.5,1])^{10}$, a hypercuboid in $\mathbf{R}^{30}$.

\subsection{The Pig Growth Model}

The pig growth model, from DeLange [1], simulates the growth of a pig with initial chemical body composition and growth parameters as defined above, under a given feeding strategy. The critical outputs after each day of simulated growth are backfat thickness and carcass weight.

A linear program is used to find the minimum cost diets for the given feeding strategy. The constitution of these diets is then used in simulating the growth of the pig.

A sample linear program is illustrated in Table 1. Diet cost per kilogram is minimised, subject to the $d$ and $r$ values in the $(d, r, p)$ triple. Parameter $d$ is incorporated in an equality constraint for the digestible energy total. Together with parameter $r$ it also dictates the minimum lysine level in the diet. The other amino acids are constrained to be present in at least certain proportions to lysine, to ensure ideal protein balance. Parameter $p$ and the ad libitum digestible energy intake curve then determine the total feed intake and so the minimum daily feed cost.

\begin{tabular}{|c|c|c|c|c|c|c|c|c|c|}
\hline & Barley & & Soyabean & & $\ldots$ & & and Bone & & \\
\hline minimise: & $0.25 \mathrm{~B}$ & + & $0.715 \mathrm{~S}$ & + & $\ldots$ & + & $0.50 \mathrm{M}$ & & \\
\hline Digestible energy & $13.20 \mathrm{~B}$ & + & $15.860 \mathrm{~S}$ & + & $\ldots$ & + & $12.50 \mathrm{M}$ & $=$ & $d$ \\
\hline Lysine lower bound & 3.19B & + & $27.330 \mathrm{~S}$ & + & ... & + & 16.19M & $\geq$ & $d r$ \\
\hline Balanced amino acid & $2.85 \mathrm{~B}$ & + & $10.740 \mathrm{~S}$ & + & ... & + & $20.30 \mathrm{M}$ & $\geq$ & $0.62 d r$ \\
\hline lower bounds & $1.20 \mathrm{~B}$ & + & $4.680 \mathrm{~S}$ & + & $\ldots$ & + & $1.09 \mathrm{M}$ & $\geq$ & $0.19 d r$ \\
\hline Mineral bounds & $0.50 \mathrm{~B}$ & + & $3.000 \mathrm{~S}$ & + & $\begin{array}{l}\cdots \\
\ldots\end{array}$ & + & $105.00 \mathrm{M}$ & $\geq$ & 8 \\
\hline 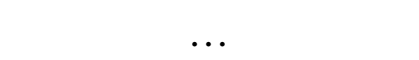 & $0.50 \mathrm{~B}$ & + & $3.000 \mathrm{~S}$ & + & $\ldots$ & + & $105.00 \mathrm{M}$ & $\leq$ & 13 \\
\hline$\cdots$ & $3.10 \mathrm{~B}$ & + & $2.600 \mathrm{~S}$ & + & $\ldots$ & + & $52.00 \mathrm{M}$ & $\geq$ & 7 \\
\hline$\cdots$ & $3.10 \mathrm{~B}$ & + & $2.600 \mathrm{~S}$ & + & $\ldots$ & + & $52.00 \mathrm{M}$ & $\leq$ & 11 \\
\hline$\ldots$ & $\ldots$ & & & & $\ldots$ & & .. & & \\
\hline Ingredient upper bounds & $-0.40 \mathrm{~B}$ & + & $0.600 \mathrm{~S}$ & - & $\ldots$ & - & $0.40 \mathrm{M}$ & $\geq$ & 0 \\
\hline$\ldots$ & $-0.10 \mathrm{~B}$ & - & $0.100 \mathrm{~S}$ & - & $\ldots$ & - & $0.10 \mathrm{M}$ & $\geq$ & 0 \\
\hline $\begin{array}{c}\cdots \\
\text { Diet mass }\end{array}$ & $\cdots_{\mathrm{B}} \mathrm{p}_{\mathrm{a}}$ & & $\cdots$ & & $\cdots$ & & $\cdots$ & & $\cdots$ \\
\hline Dlet mass & B & + & 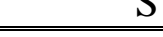 & + & ... & + & 101 & $=$ & 1 \\
\hline
\end{tabular}

Table 1. Linear program providing the least cost diet. 
For a given feeding strategy, the model allows calculation of total feed costs for the pig, as well as the final backfat thickness and carcass weight. These outputs will be used to evaluate the objective function.

\subsection{The Objective Function}

Pig farmers wish to maximise gross margin per pig place per year, namely

(Gross Return - Total Feed Costs -Weaner Cost) per pig place per year

The gross return to the farmer is determined by the price schedule for pigs at slaughter, which typically depends on backfat thickness and carcass weight. A 1998 New Zealand schedule is reproduced in Table 2. For pigs in the best categories (backfat thickness from $6 \mathrm{~mm}$ to $9 \mathrm{~mm}$ and carcass weight between $50 \mathrm{~kg}$ and $55 \mathrm{~kg}$ ) the producer receives $\mathrm{NZ} \$ 3.10 / \mathrm{kg}$.

\begin{tabular}{|c|c|c|c|c|c|c|c|c|c|c|c|}
\hline \multirow{4}{*}{ Fat (mm) } & \multicolumn{11}{|c|}{ Weight $(\mathrm{kg})$} \\
\hline & 35.0 & 35.1 & 40.1 & 45.1 & 50.1 & 55.1 & 60.1 & 65.1 & 70.1 & 75.1 & 80.1 \\
\hline & and & to & to & to & to & to & to & to & to & to & and \\
\hline & unde & 40.0 & 45.0 & 50.0 & 55.0 & 60.0 & 65.0 & 70.0 & 75.0 & 80.0 & over \\
\hline Under 6 & 250 & 250 & 250 & 250 & 250 & 250 & 250 & 250 & 250 & 250 & 250 \\
\hline & 310 & 310 & 310 & 310 & 310 & 295 & 295 & 280 & 280 & 280 & 275 \\
\hline 10 to 12 & 285 & 285 & 285 & 285 & 285 & 280 & 280 & 280 & 280 & 280 & 275 \\
\hline 13 to 15 & 190 & 190 & 190 & 215 & 215 & 245 & 245 & 245 & 245 & 245 & 245 \\
\hline 16 to 18 & 150 & 150 & 150 & 150 & 150 & 165 & 165 & 165 & 165 & 165 & 165 \\
\hline Over 18 & 120 & 120 & 120 & 120 & 120 & 135 & 135 & 135 & 135 & 135 & 135 \\
\hline
\end{tabular}

Table 2. New Zealand price schedule giving prices in cents per $\mathrm{kg}$ for pigs at slaughter, as at 24 August 1998. $\$ 9.40$ per carcass is deducted for levies.

The objective function of interest is $g(F)$, the gross margin per pig place per year associated with feeding strategy $F$. This is calculated as $\max _{x} g(F, x)$, where $g(F, x)$ is the gross margin per pig place per year when feeding strategy $F$ is administered for $x$ days, $x \leq 70$.

In the first step, the gross margin per pig place per year is calculated for a given feeding strategy $F$ and number of days $x \leq 70$ for which it is fed, as detailed in Figure 3. 


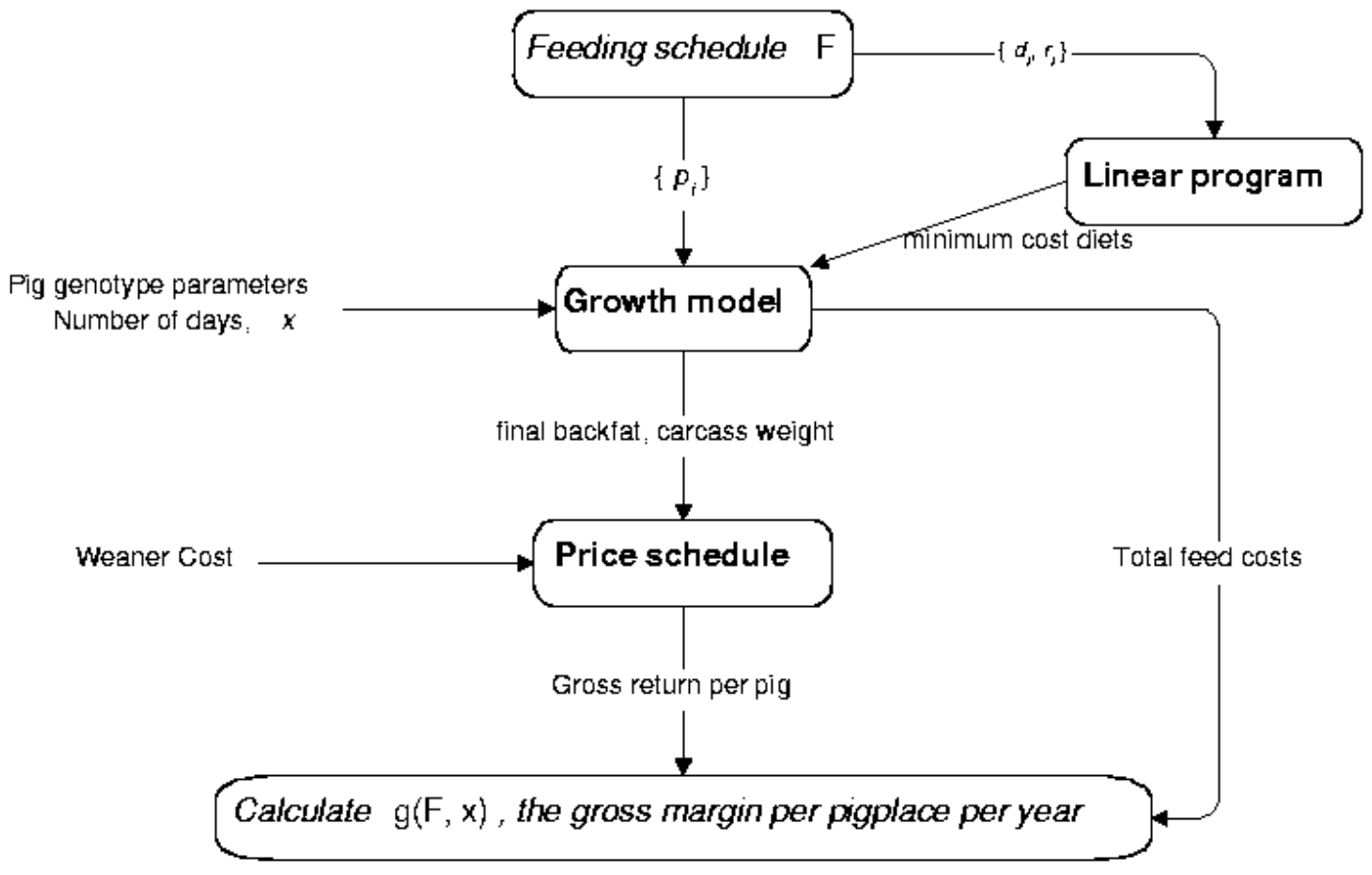

Figure 3. Flow chart for calculation of $g(F, x)$.

A linear program finds the minimum cost diets for the given feeding strategy. The pig growth model and price schedule are then used to find the market return per pig, from which the gross margin per pig place per year $g(F, x)$ may be calculated.

Finally, $g(F)$ is found as the maximum in the array $(g(F, 1), g(F, 2), \ldots, g(F, 70))$.

To summarise: a linear program and a pig growth model allow evaluation of the gross margin per pig place per year for any feeding strategy. The objective function is now maximised over a high dimensional hypercuboid, using a non-linear program.

\section{Solution Algorithms}

Complexities of the pig growth model and the use of linear programming and maximisation over $x$ mean that the objective function is of the "black box" variety; it is not known analytically. Solution algorithms thus require many objective function evaluations, involving substantial computation. The domain is also of moderately high dimension.

Simulated annealing [3] and genetic algorithms [2] are both successful stochastic approaches to the problem, while a satisfactory deterministic approach is provided by the Nelder-Mead algorithm [4]. They typically yield, for the particular problem used, a gross margin of around NZ $\$ 280$ per pig place per year. Pure random search, however, is not successful. Figure 4 shows the progress of a genetic algorithm on the problem, compared with that of Pure Random Search. In 30 seconds a genetic algorithm can find a feeding strategy superior to that reached by pure random search in over 100 hours! Pure random search, in that time, reaches a solution of approximately NZ $\$ 250$ per pig place per year. 


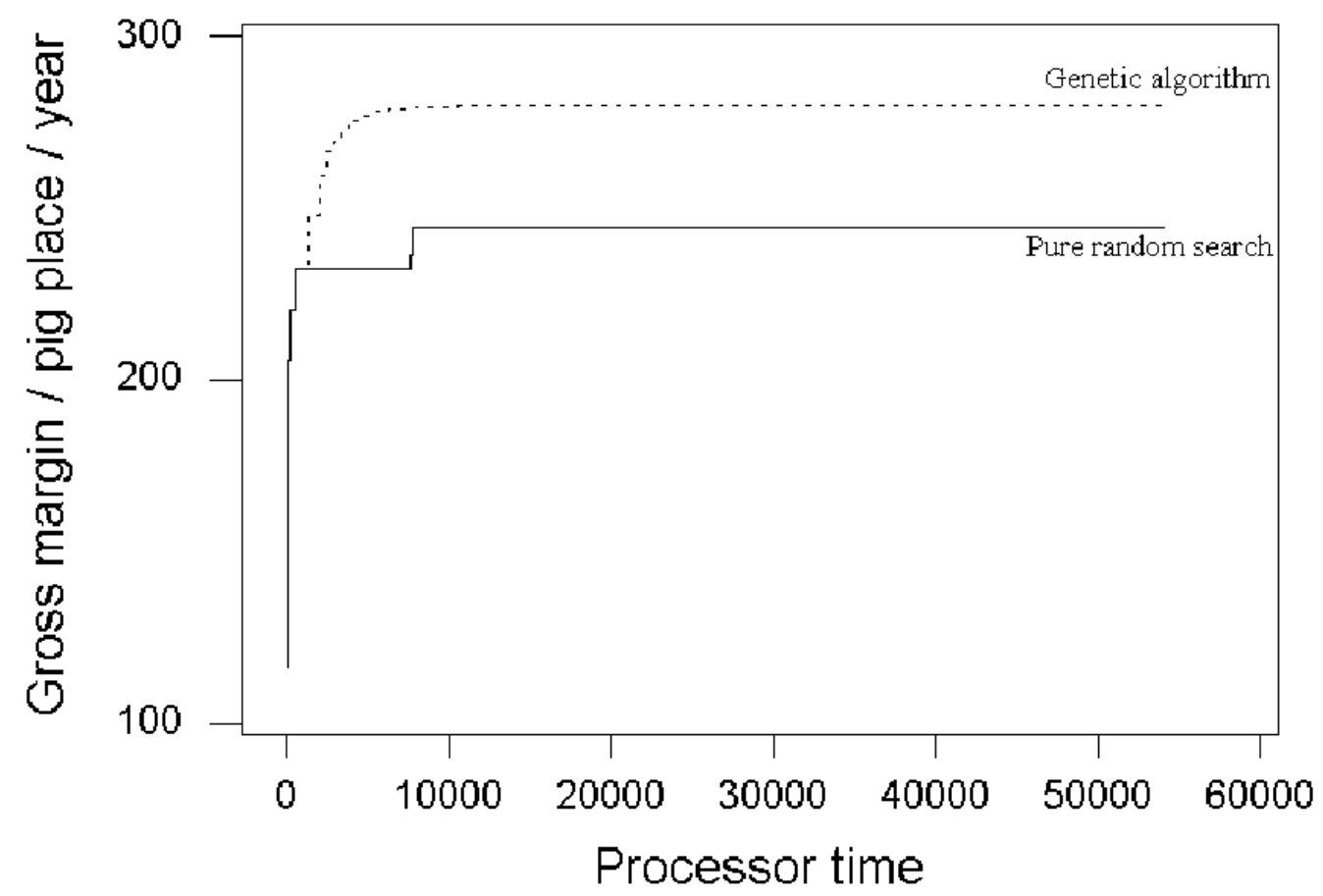

Figure 4. Comparison of a genetic algorithm and pure random search, in maximising the gross return per pig place per year.

Table 5 displays the final results of a successful run. It lists the values of $d, r$ and $p$ in the best solution; the cost in cents per kilogram of each diet is also given. Slaughter date $x$ was selected as 63 days, so the parameters for the tenth week were not used and are not shown.

\begin{tabular}{ccccc} 
Week & $d$ & $r$ & $p$ & Cost $(\mathrm{c} / \mathrm{kg})$ \\
\hline 1 & 14.51 & 0.791 & 0.961 & 41.24 \\
2 & 14.57 & 0.627 & 1.000 & 35.93 \\
3 & 14.40 & 0.554 & 1.000 & 33.10 \\
4 & 13.14 & 0.568 & 0.840 & 30.61 \\
5 & 14.34 & 0.493 & 0.873 & 31.00 \\
6 & 14.18 & 0.478 & 0.850 & 30.20 \\
7 & 13.58 & 0.758 & 0.510 & 37.55 \\
8 & 13.14 & 0.550 & 0.670 & 30.07 \\
9 & 14.14 & 0.528 & 0.680 & 31.66 \\
\hline \hline
\end{tabular}

Table 5. Weekly values of $d, r$ and $p$ and cost per kilogram of diet for a solution found using a genetic algorithm.

The linear program then provides the ingredients for each diet in the feeding strategy, together with the optimal proportion of each ingredient by weight. For example, a kilogram of the first diet in table 3 contains $510 \mathrm{~g}$ barley, $280 \mathrm{~g}$ soyabean and $100 \mathrm{~g}$ wheat by-product, with the remainder made up of meat and bone meal, soya oil, salt and synthetic amino acids.

In practice, feeding regimens cannot be administered precisely. Allowing for a practicable tolerance of 0.05 in each parameter of each diet, however, it can be seen that the optimal solution is still only one part of the defined solution space in $10^{40}$. An intelligent search method is needed in order to find solutions approaching this optimum, as comparison with pure random search has shown. Even with only three distinct diets (as is common in practice today), the optimal solution is one part in $10^{12}$. A useful, 
practical solution region remains a needle in a high dimensional haystack. As pig producers move toward continuous feeding, the number of distinct diets available for the pig, and thus the dimension of the problem, will increase. The need for intelligent optimisation methods will become even more pressing.

\section{The Nature of the Objective Function}

A question that always vexes the global optimiser is whether the overall optimum has been reached. In order to investigate this question in this context, further information about the nature of the objective function is obtained in two steps:

1. The value of the objective function along randomly taken domain cross-sections through the best known solution is examined. This provides evidence that a solution close to the optimum has been reached, and suggests that the objective function takes the shape of a single peaked, but very craggy, high-dimensional volcano.

2. The performance of pure random search is compared with the expected performance if the form was broadly that of this "craggy volcano". The observed agreement between the two provides support for the hypothesised objective function shape.

\subsection{Cross-section}

Figure 6 shows the typical shape of the objective function along a random cross-section through the putative argmax. Overall, the function appears to be unimodal. Jumps in the function are caused by the step function nature of the price schedule and the fact that the pig must grow a whole number of days.

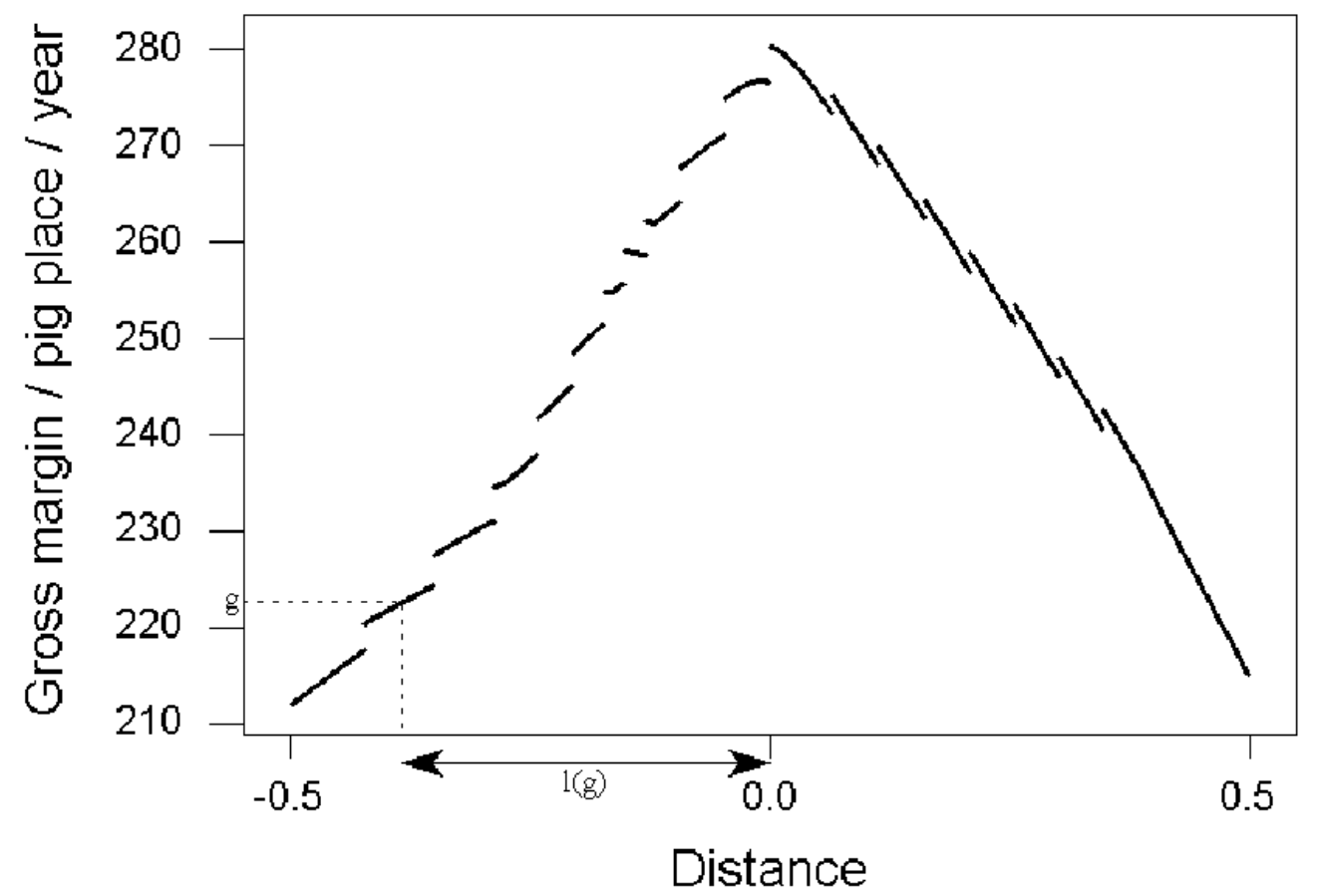

Figure 6. The approximately volcano shape of a section through the objective function at the best known solution. 
Through examination of many such cross-sections, an average relationship can be set up between $g$, the gross margin per pig place per year, and $l(g)$, the distance between the corresponding place in the domain and the best known solution (as illustrated in Figure 6). The expected number of iterations until convergence to a particular level for pure random search will be the reciprocal of the probability that a given iteration falls in the associated level set. This probability is the relative volume of the level set. Assuming roughly hyper-spherical level sets for the objective function, the probability is

$$
\frac{\pi^{15} l(g)^{30}}{15 ! V}
$$

where $V$ is the volume of the hypercuboid domain. By estimating $l(g)$, the expected performance of pure random search on the problem can be predicted.

\subsection{Pure Random Search Comparison}

Figure 7 compares this theoretical expected number of pure random search iterations to reach a given gross margin per pig place per year with a step function recording progress of a particular run.

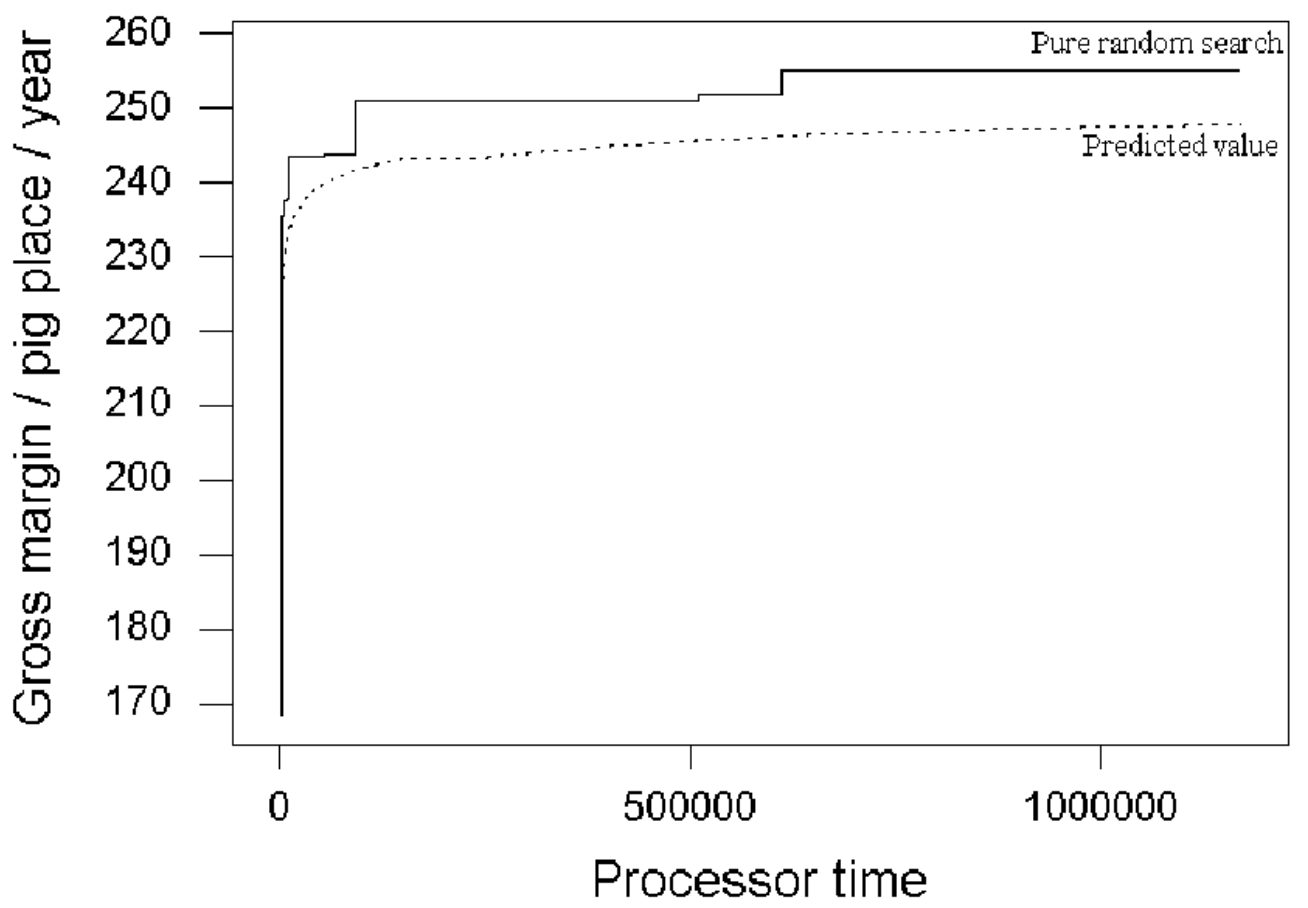

Figure 7. Comparison of the predicted time to reach a given value (dotted line) with the time required in a particular run of pure random search (solid line).

Evidently the forms of the two curves are indeed similar. However, actual runs tend (as depicted in Figure 7) to be slightly higher than the predicted curve calculated based on the estimated relationship between $g$ and $l(g)$, assuming hyperspherical level sets. This may well indicate that smaller peaks exist. There are also some cross-sections where the objective function does not decrease very rapidly. Overall there is evidence that the level sets have slightly greater relative volume than predicted.

However, the general agreement in shape provides support for the form of the postulated relationship between $g$ and $l(g)$, and for the suggestion that the objective 
function has only one significant peak. This tends to confirm that the solution found by use of the search algorithms is indeed close to the overall optimum. If, as appears possible, the assumptions concerning objective function shape are broadly true, then a theoretical explanation for the poor performance of pure random search is also provided.

\section{Summary}

This paper describes a fairly complicated problem, which has for many years eluded solution. The development of pig growth models, together with the availability of nonlinear optimisation tools on high speed computers, has now made it possible to extend the traditional use of optimisation in pig nutrition. Linear programming to determine least cost diets is used as a component of a larger optimisation routine, by which the challenge of diet formulation to maximise gross return per pig place per year can be answered.

The solution methods used are demonstrated to be significantly superior to pure random search. Optimisation methods show considerable promise for increasing the efficiency of the worldwide industry of pig farming.

\section{Acknowledgements}

Professor Paul Moughan is thanked for his encouragement and pertinent questions during the development of this material at Massey University. The contributions of Lindsay Alexander in providing programming advice and of Christopher Clark in developing relevant software is also much appreciated.

\section{References}

[1] C.F.M. DeLange, Framework for a simplified model to demonstrate principles of nutrient partitioning for growth in the pig, Modelling Growth in the Pig, EAAP Publication 78 (P.J. Moughan, M.W.A. Verstegen and M.I. Visser-Reyneveld, (eds)), (1995), pp 71--85

[2] J.H. Holland, Adaptation in Natural and Artificial Systems, University of Michigan Press, Ann Arbor, MI., (1975)

[3] S. Kirkpatrick, C.D. Gelatt Jr., M.P. Vecchi, Optimisation by Simulated Annealing, Science, 220, 4598 (1983), pp 671--680

[4] J.A. Melder, R. Mead, A simplex method for function minimisation, The Computer Journal, 7 (1965), pp 308--313 